\title{
Evidence for time-based models of free recall
}

\author{
GORDON D. A. BROWN and CAROLINE MORIN \\ University of Warwick, Coventry, England \\ and \\ STEPHAN LEWANDOWSKY \\ University of Western Australia, Nedlands, Western Australia, Australia
}

\begin{abstract}
Is memory temporally organized? According to temporal distinctiveness models of memory, temporally isolated items should be better remembered than temporally crowded items in free recall tasks. Here, we tested this class of model by varying the temporal isolation of items either predictably (Experiment 1) or unpredictably (Experiment 2) in a free recall task. In both experiments, item recall probability increased as a function of the temporal gaps both before and after the item. The results are taken as support for temporal distinctiveness models of memory, in which items are represented and recalled in terms of their positions along a temporal dimension.
\end{abstract}

The suggestion that items might be organized in memory along a temporal dimension, so that items that are presented in temporal proximity will be relatively confusable and hard to retrieve, is embodied explicitly in a number of recent temporal distinctiveness models of memory (e.g., Brown, Neath, \& Chater, 2002; Brown, Preece, \& Hulme, 2000; Neath, 1993) and is implicit in many others. An intuitive foundation for such models is given by the well-known telephone pole analogy (Bjork \& Whitten, 1974; Crowder, 1976), according to which memories become less discriminable from one another (and hence, less retrievable) as they recede into the temporal distance, just as evenly spaced telephone poles will become less visually distinctive to a stationary observer as they recede into the spatial distance. This analogy motivates models in which memories are represented in memory at least partly in terms of their temporal distance from the present. If it is assumed that (1) the temporal distance dimension is logarithmically compressed, so that temporally distant items are more confusable, and (2) items occupying nearby locations on the dimension are less discriminable in memory, such an account can be implemented (e.g., Brown et al., 2002) and shown to account for a number of memory phenomena, such as recency effects and their interactions with retention interval (Bjork \& Whitten, 1974; Nairne, Neath, Serra, \& Byun, 1997; although see Davelaar, Goshen-Gottstein, Ashkenazi, Haarmann, \& Usher, 2005).

A key prediction is that items that are temporally isolated at learning will be easily discriminable from their

This research was supported by ESRC Grant RES 000231038 to the first and second authors and by a Linkage International Grant from the Australian Research Council to the first and third authors. Address correspondence to G. D. A. Brown, Department of Psychology, University of Warwick, Coventry CV4 7AL, England (e-mail: g.d.a.brown@warwick .ac.uk). immediate neighbors and, hence, relatively well recalled. This is predicted by the same mechanism that enables temporal distinctiveness models to account for recency effects (cf. the telephone pole analogy). Thus, the mechanism that predicts extended recency effects also predicts effects of temporal isolation at presentation, and so any experimental task that produces substantial recency effects should (according to temporal distinctiveness models) also give rise to presentation-induced temporal isolation effects.

\section{Existing Data}

We first will review temporal isolation effects in serial recall, since recent results appear hard to reconcile with temporal distinctiveness models of memory for serial order.

Serial recall. Welte and Laughery (1971) examined serial recall following increasing or decreasing presentation rates of nine-digit lists and found that items were less well recalled in regions of the list where presentation rate was relatively fast (i.e., late in the list for a decreasinggaps schedule). However, the effect was absent in an order reconstruction task (see also Corballis, 1966b). Neath and Crowder (1996) examined serial recall of five-item lists, using increasing and decreasing schedules (in addition to a control regular presentation rate) and fast presentation to minimize rehearsal. Like Welte and Laughery, they found that temporally crowded items were less well recalled. At first blush, these results appeared to support temporal distinctiveness models of serial order. However, subsequent experiments have shown that temporal isolation effects are seen in serial recall only when the presentation schedule is predictable (as in the Welte \& Laughery and Neath $\&$ Crowder experiments). When the temporal isolation of items is randomized within each to-be-remembered sequence (Corballis, 1966a) and rehearsal during interitem gaps is prevented, serial recall for an item is no better if that item is temporally isolated (Lewandowsky \& Brown, 
2005; Lewandowsky, Brown, Wright, \& Nimmo, 2006). This absence of a temporal isolation effect in serial recall is found when presentation is auditory (Nimmo \& Lewandowsky, in press), when interitem gaps are large (Nimmo \& Lewandowsky, 2005), and when a probed recall task is used (Lewandowsky et al., 2006). Lewandowsky et al. concluded that unmodified temporal distinctiveness models of serial recall (e.g., Brown et al., 2000) are incorrect, at least when presentation schedule is unpredictable, and must be extended to incorporate a positional dimension (Henson, 1999; Ng \& Maybery, 2002).

Free recall. Temporal isolation effects might be expected to emerge reliably in free recall, in contrast to serial recall, for at least two reasons. First, the signature of temporal distinctiveness models, and of the telegraph pole analogy generally, is a substantial and extended recency effect when recall is immediate. Such recency effects typically do not emerge in forward serial tasks of the type reviewed above. Models could, therefore, abandon the application of temporal distinctiveness to serial recall, while preserving a time-based explanation of recency effects in free recall tasks. However, given that temporal distinctiveness models take much support from recency effects, such models would be severely challenged if strong effects of recency, but not of temporal isolation, appeared in the same experiment. We are unaware of any such data. Although Lewandowsky et al. (2006) found some recency but no effect of temporal isolation in a partialreport probed recall task, the amount of recency was small, in comparison with that typically observed in free recall or positional probe recall tasks, with performance below $70 \%$ correct even for the final serial position.

A second consideration concerns the differing task demands of serial and free recall. A serial recall task highlights the positional, rather than the temporal, dimension; the task is explicitly to recall the first item, then the second item, and so on. There may, therefore, be little or no advantage in, and perhaps a cost to, encoding order with such precision in free recall or recognition tasks. More specifically, free recall and recognition tasks do not require the recovery of positional information for correct performance but, instead, can be seen as tapping memory for items that occur within a temporal window defined by the to-be-remembered list. It would, therefore, be unsurprising if free recall and serial recall encouraged differential emphasis on temporal and positional dimensions, respectively.

Examination of temporal isolation effects in a traditional free recall paradigm therefore provides a crucial test case. Unfortunately, existing data are scant, and the results that do exist are open to alternative, nontemporal explanations and/or are obtained using much shorter lists than in traditional free recall. Neath and Crowder (1990) used increasing and decreasing schedules of presentation and examined free recall for five-item lists of letters or word pairs. The results were consistent with the idea that temporally crowded items are less well recalled, although clear patterns independent of recency effects (which formed the focus of the Neath \& Crowder study) are difficult to discern. Glenberg and Swanson (1986) found that increasing the temporal gap before the last of five word pairs improved memory for that pair under auditory, but not visual, presentation. Rönnberg (1980) examined free recall of 10-word lists following increasing, decreasing, constant, or random presentation schedules. When list length was constant and a standard free recall procedure was used, there was a clear tendency for items in the more temporally crowded regions of the lists to be less well recalled (see also Rönnberg, 1981). The interaction was somewhat clearer when overall presentation rate was fast, rather than slow, consistent with the possibility that rehearsal, which was not prevented in the Rönnberg study, may serve to mask temporal isolation effects.

Although various aspects of these studies (e.g., the use of short lists or the failure to prevent rehearsal) inhibit any definitive conclusion on temporal isolation effects in free recall, one crucial aspect of all the studies in which gap size effects have been analyzed is that presentation consistently either increased or decreased throughout a list. Strategic effects may, therefore, be present, as in serial recall. In the first experiment below, therefore, we examined temporal isolation effects in free recall of lists, using either increasing or decreasing schedules of presentation when rehearsal was prevented; in the second experiment, temporally random presentation was used.

\section{EXPERIMENT 1}

In Experiment 1, we examined the effects of temporal isolation when (1) rehearsal was prevented and (2) longer lists of items, typical of those used in traditional free recall paradigms, were used. A free recall paradigm was used, with a continuous distractor task (rapid reading aloud of digits in the intervals between the words to be remembered) to prevent rehearsal. Predictable schedules of either an increasing or a decreasing presentation rate were used.

\section{Method}

Participants. Seventeen 1st-year students from the University of Warwick participated for course credit. Four participants were replaced due to poor performance (less than $30 \%$ correct recall overall), in order to ensure sufficient analyzable data from the middle portion of the serial position curve.

Stimuli. Three hundred forty one-syllable nouns of four to six letters and with a written word frequency between 10 and 50 words per million were taken from the MRC Psycholinguistic Database (Wilson, 1988). Twenty lists of 17 words were presented. The first four lists served as practice trials, leaving 16 experimental trials. Eight were from the decreasing condition; 8 were from the increasing condition. The trials from the two conditions were presented in random order.

Procedure. The participants were tested individually in a soundattenuating booth with the experimenter present. In the decreasing condition, the number of digits presented between the items was as follows: 7, 7, 6, 6, 5, 5, 4, 4, 3, 3, 2, 2, 1, 1, 0, and 0 . The reverse sequence was used for the increasing condition. Words were presented for $700 \mathrm{msec}$ ( $500 \mathrm{msec}$ on, $200 \mathrm{msec}$ off), whereas digits were presented for $500 \mathrm{msec}$ ( $350 \mathrm{msec}$ on, $150 \mathrm{msec}$ off). A 150msec blank screen and then a recall signal followed the last item. The participants had 1 min to recall orally as many words as they could, regardless of order. 


\section{Results}

The responses were scored as correct if the item was recalled from the last list presented. To illustrate the effects of temporal crowding independently of elapsed time, Figure 1 shows the mean proportion of item recalled for each schedule as a function of temporal recency of presentation at the start of recall. (This method was used because the time between an item's presentation and the start of recall is confounded with condition. For example, the ninth [middle] item was closer in time to the recall signal in the decreasing condition than in the increasing condition.) It is clear that temporal crowdedness exerts a strong effect over and above presentation-recall delay, so that early-list items were better remembered in the decreasing condition, whereas the reverse was the case for late-list items (with the exception of very recently presented items, which were well recalled irrespective of presentation schedule). These results appear to be consistent with the predictions of temporal distinctiveness models.

Conventional statistical analysis was undertaken using serial position as a factor. The first three and last five serial positions were excluded from the analysis as buffer items. (Adoption of this conservative procedure was motivated partly by the assumption of several extant models that edge effects may give rise to small serial position effects for end items, partly to avoid ceiling effects for recency items, and partly to exclude possible interpretations of any interaction in terms of a separate time- or item-limited short-term store.)

A $2 \times 9$ (schedule of presentation $\times$ serial position) ANOVA revealed no overall effect of the schedule of presentation $\left[F(1,12)=2.71, M S_{\mathrm{e}}=0.017, p=.13\right]$ and no significant effect of serial position $\left[F(8,96)=1.77, M S_{\mathrm{e}}=\right.$ $0.017, p=.10]$, but a significant interaction $[F(8,96)=$ $\left.2.34, M S_{\mathrm{e}}=0.024, p=.03\right]$.

The gap between the first two items predicted the duration of the following gaps, leading to the possibility that strategic effects occurred at encoding. We therefore asked the participants at the end of the experiment if they had been aware that the presentation schedule was always either increasing or decreasing. Nine participants had not noticed a difference between the two schedules, whereas the other four had. An analysis revealed no significant difference between the two groups. Indeed, an examination of the mean probability of recall for the first five serial positions reveals that the difference between the increasing and the decreasing schedules was larger for the participants who were unaware (aware, .16 for increasing, .30 for decreasing; unaware, .19 for increasing, .44 for decreasing). Thus, the results seem difficult to explain in terms of different encoding strategies.

A final issue concerns the possibility of output order effects. Recall is not instantaneous, and the true time that elapses between presentation and recall of a given item will, therefore, depend on the time course and order of recall. We therefore examined recall timing and order as a function of condition (increasing vs. decreasing). First, the time to initiate recall was similar for the increasing $(1.6 \mathrm{sec})$ and the decreasing $(1.3 \mathrm{sec})$ conditions. The difference, although reliable $[t(12)=6.5, p<.001]$, was very small, relative to the temporal variation between conditions (cf. Figure 1). Furthermore, in both conditions, more than half the recalled items were output within the subsequent $4 \mathrm{sec}$. Thus, the time course of recall was sufficiently short that variations in the temporal protocol of output across conditions could not significantly distort the observed interaction. Potentially more important is the order of recall. Recall order effects could contribute to the observed interaction if, for example, early-list items are recalled earlier in the decreasing condition than in the increasing condition, leading to superior recall for those items because of their earlier recall, rather than (or as well as) their greater temporal isolation. Analysis revealed that, if anything, the reverse was the case; Figure 2 shows the

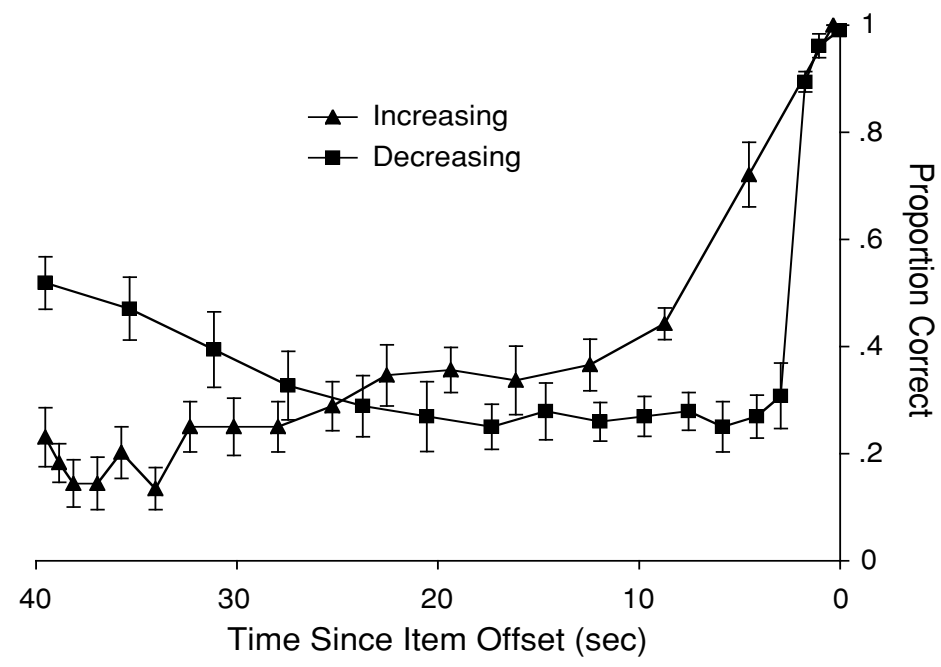

Figure 1. Serial position curves for the increasing and decreasing schedule of presentation as a function of the time since item offset in Experiment 1. 

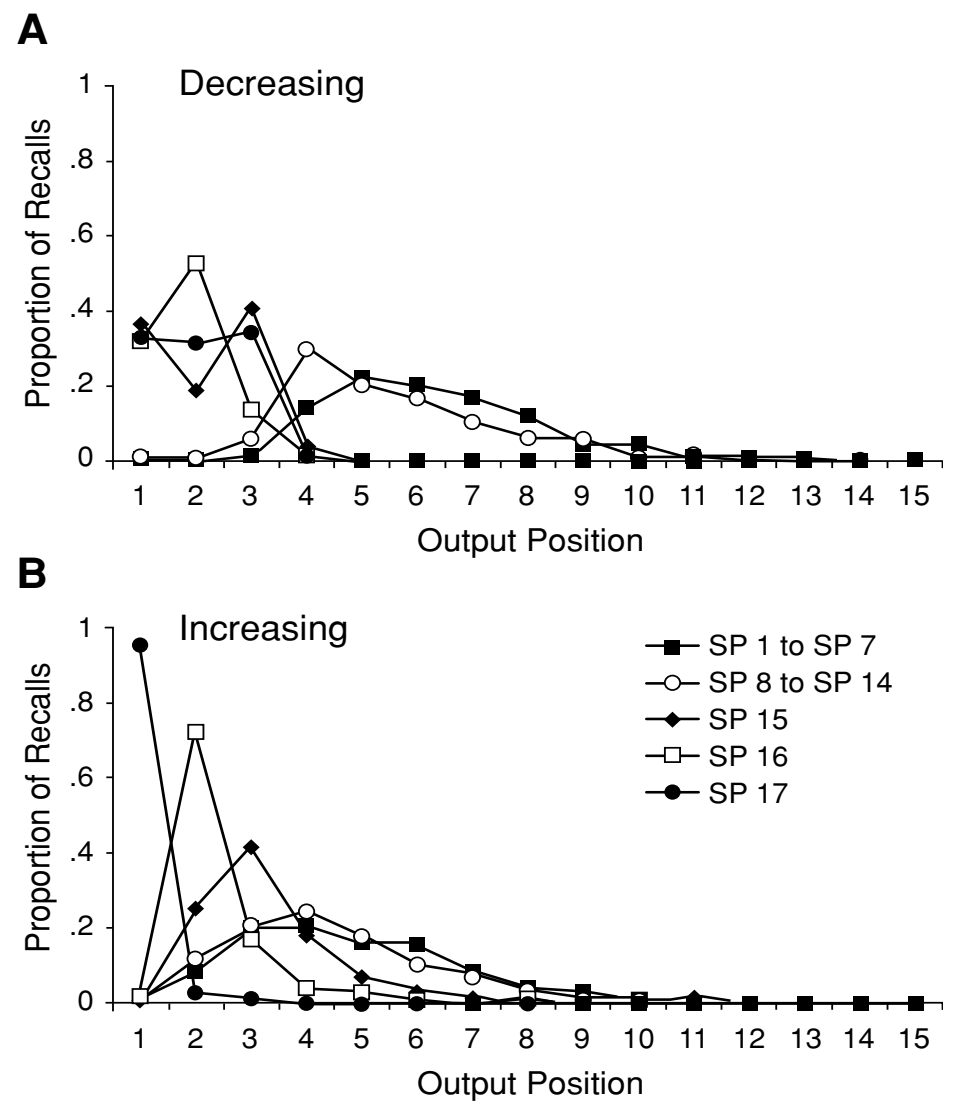

Figure 2. Position of recall for (A) the decreasing gaps condition and (B) the increasing gaps condition. Each line shows, for a given item (or group of items), the proportion of time that item (or items) was recalled in each output position. SP, serial position of presentation.

output recall positions separately for each of the most recent 3 items, for the 8 th through 14 th items, and for the 1 st through 7 th items. In general, the most temporally recent items were recalled first, irrespective of their crowdedness. Thus, in the increasing-gaps condition, only the last item was very temporally recent, and it was almost invariably the first to be recalled. In the decreasing-gaps condition, in contrast, the last few items were all very temporally recent, and each of them had a good chance of being recalled in any of the first three output positions. This is, of course, just what a temporal distinctiveness model would predict; temporally recent items will benefit most from their recency to the extent that they are recalled after a short interval (before their relative distinctiveness declines). Items presented in the first seven positions were recalled slightly earlier in the increasing condition (mean output position, 4.8 ) than in the decreasing condition (mean output position, 6.3), allowing us to exclude the possibility that the superior recall of early-presented items in the decreasing condition reflected their earlier recall.

In summary, recall order effects cannot give rise to the observed temporal isolation effects. Output order differences primarily affect only very recent items, and these are recalled close to ceiling in any case.

\section{Discussion}

The results of Experiment 1 show clear effects of temporal isolation. They extend the results of previous studies that have typically used shorter lists and/or failed to prevent rehearsal. In general terms, the results therefore appear to be consistent with the predictions of temporal distinctiveness models. However, nontemporal models might be able to explain the results if it is assumed that some form of time-based trace consolidation occurs in the temporal gaps following items. It seems unwise to assume that all such processes could be prevented by the continuous distractor methodology that was used. Furthermore, as was noted earlier, studies of serial recall have shown that the effects of temporal isolation disappear when schedules are not predictable.

In Experiment 2, we therefore again examined free recall and used the same set of preitem and postitem gaps. However, interitem gaps were chosen randomly for each list.

\section{EXPERIMENT 2}

\section{Method}

Participants and Apparatus. Twenty-two 1st-year students from the University of Warwick participated voluntarily in exchange 


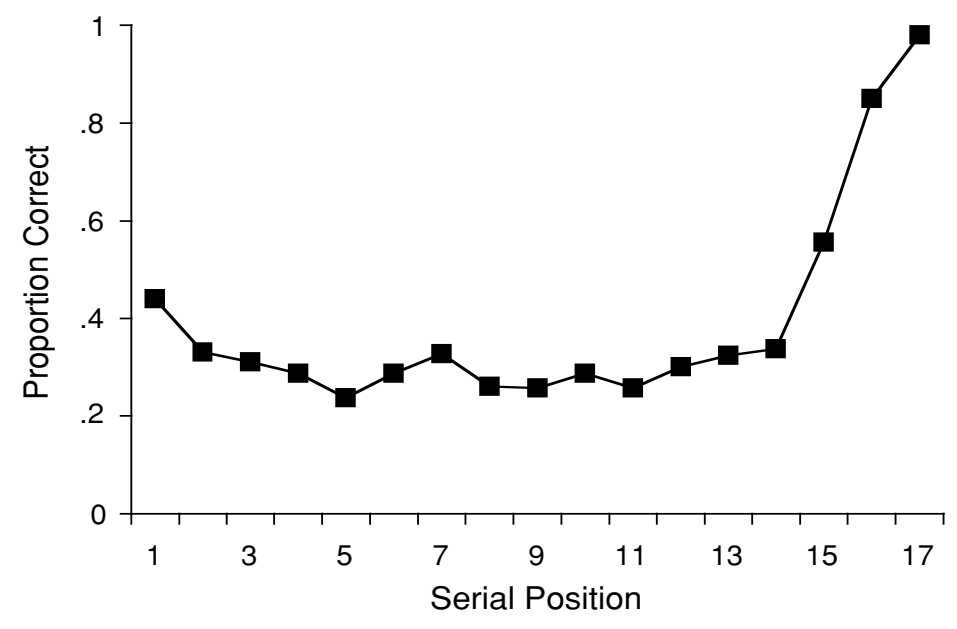

Figure 3. Serial position curve regardless of the temporal gaps before and after each item in Experiment 2.

for course credit. Six participants were replaced due to poor performance (less than $30 \%$ of correct recall overall). One participant was replaced due to exhibiting a bizarre pattern of results in which he recalled only 2, 3, or 4 items from several of the early lists but then apparently adopted a dramatically different strategy and recalled 12 and 14 items from the last two lists.

Stimuli and Procedure. Experiment 2 was identical to Experiment 1 in all respects, except that a random order of gaps was used. The same digit-filled gaps were used for the continuous distractor task but were presented randomly within the list. The overall time taken to present a list was thus constant and identical in both experiments.

\section{Results}

Figure 3 shows the overall serial position curve produced (averaged over all presentation schedules); it is evident that there was strong recency and small primacy, as is expected when rehearsal is prevented (e.g., Tan \& Ward, 2000).

Of more interest is the effect of temporal isolation. As in Experiment 1, we ignored data from primacy (three items) and recency (five items) buffers. We first examined the effects of total temporal isolation (the sum of pre- and postitem gaps) in item recall. To aid independence of observations, we looked only at performance on alternating serial positions (Items 4, 6, 8, 10, and 12). There are inevitably fewer observations for extreme total isolations (because there are few occasions when an item is both preceded and followed by a very short or long gap).

There are 15 possible total temporal isolations, with the shortest being $0 \mathrm{sec}$ and the longest being $7 \mathrm{sec}$. We collapsed these into seven pairs of adjacent temporal isolations, ignoring the longest value. Proportion of recall is shown as a function of total temporal isolation in Figure 4. Recall probability increased with total temporal isolation, rising from around $17 \%$ correct (when total temporal isolation is $<1 \mathrm{sec}$ ) to around $35 \%$ (when total temporal isolation is $>6 \mathrm{sec}$ ).

The strong effect of temporal isolation is consistent with the predictions of temporal distinctiveness models. Next, we examined the key prediction, made by such accounts, of separate effects of both preitem gap and postitem gap.

To obtain sufficient data points, we used all nine serial positions within the middle portion of the curve (exclusion of primacy and recency buffers allowed us to ignore any confound between gap duration and temporal recency). Separate analyses were performed to examine the effects of preitem and postitem interval on items in those serial positions. (These analyses are not independent, because the preitem gap for item $n$ in one analysis will also be the postitem gap for item $n-1$ in the other analysis.)

Prior to analysis, it was felt important to exclude confounds that might be introduced by sequential dependencies in output. It is well established that a recall of, for example, Item $n$ is relatively likely to be followed by recall of Item $n+1$ (Kahana, 1996; Laming, 1999), and indeed, recent models have emphasized the importance of such

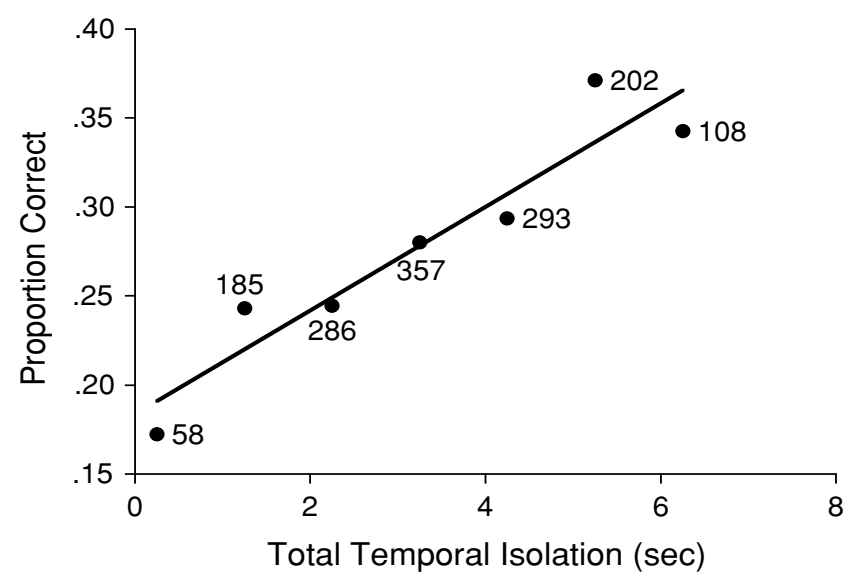

Figure 4. Proportion of recall as a function of the total temporal isolation in Experiment 2. The numbers that accompany the points give the number of observations on which each point is based. 
data (e.g., Howard \& Kahana, 1999, 2002). Such effects could lead to misleading apparent effects of preitem gap. For example, suppose that on a given trial, Item $n$ is recalled because it is followed by a large temporal gap. Item $n+1$ might then be recalled just because Item $n$ has been recalled - that is, even if there is no causal relationship between the recall of Item $n+1$ and the large temporal gap following Item $n$ and, hence, preceding Item $n+1$. Analysis might, nevertheless, note a statistical relationship between preitem gap and recall probability. To obviate this possibility as far as possible, we looked at the effects of preitem gap only in cases in which the previous item had not been recalled, and we looked at effects of postitem gap only in cases in which the following item had not been recalled. ${ }^{1}$ Figures 5A and 5B show the effects on recall probability of preitem gap and postitem gap, respectively.

It is evident that there were clear effects of both preitem gap and postitem gap and that the effects were similar in magnitude. We computed, separately for each participant, the correlation between preitem gaps and corresponding recall probability and the correlation between postitem gaps and corresponding recall probability. For the preitem gap, the mean (median) correlation was .29 (.35), and this differed from zero $[t(14)=3.45, p<.005]$. For

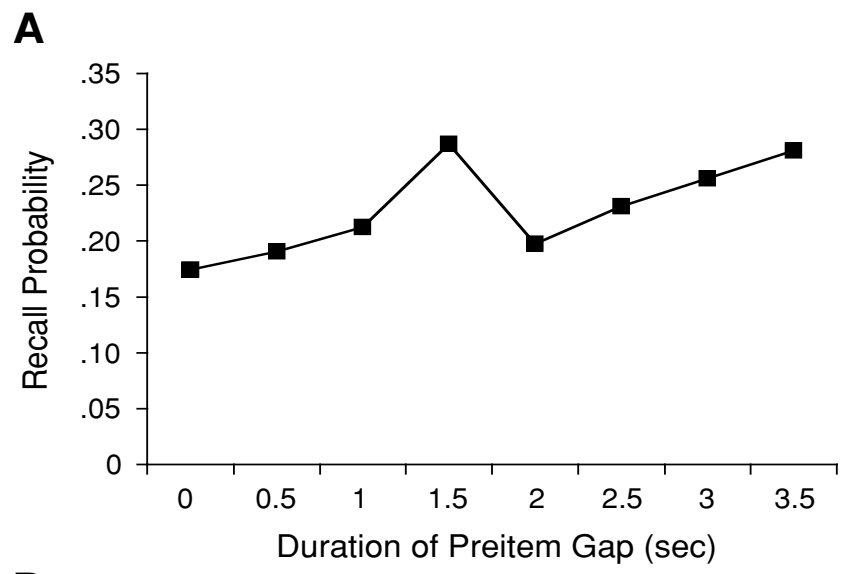

B

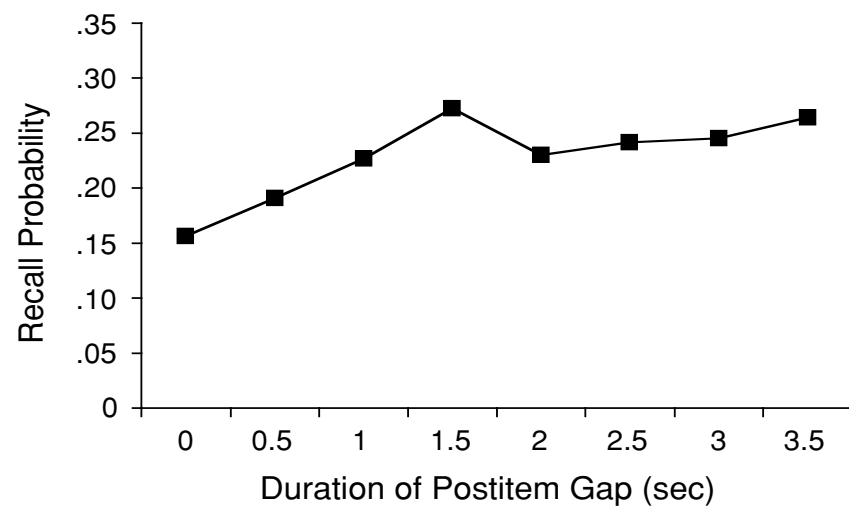

Figure 5. Proportion of recall as a function of preitem (A) and postitem (B) gaps in Experiment 2. the postitem gap, the effect was also significant: The mean (median) correlation was .27 (.17), and this differed from zero $[t(14)=2.97, p<.05]$.

We also conducted separate regression analyses for each participant and examined the regression coefficients. The pattern of results was similar to that observed in the analysis of correlations. For the effect of preitem gap, the mean (median) regression coefficient was 7.1 (8.5), and this was significantly different from zero $[t(14)=3.56$, $p<.005]$. The effect of postitem gap was also significant: The mean (median) coefficient was 5.8 (3.2), and this was significantly different from zero $[t(14)=3.11, p<.01] .^{2}$

\section{Discussion}

The results of Experiment 2 are straightforward and mirror the findings from Experiment 1, in that there was a clear effect of temporal isolation on free recall. More important, there was a clear effect of preitem gap that could not be explained by models that assume that apparent temporal isolation effects, in fact, reflect some process such as consolidation, rehearsal, or another process that could, by necessity, occur only in the gaps following an item's presentation.

\section{SUMMARY AND CONCLUSION}

Temporal distinctiveness models predict that an item's retrievability should be determined by the temporal gaps preceding and following it. In serial recall, previous research has shown effects of temporal isolation when predictable increasing or decreasing presentation schedules are used. However, such effects largely disappear when unpredictable schedules of presentation are used. In free recall, in contrast, there has been a lack of unequivocal evidence for or against temporal distinctiveness models, despite ubiquitous accounts of recency effects in terms of mechanisms involving temporal distinctiveness (although see Davelaar et al., 2005).

The results of two free recall experiments in the present article have shown clear effects of temporal isolation when presentation schedule is either predictable (Experiment 1) or unpredictable (Experiment 2). The results have a number of theoretical implications. First, in the case of serial recall, it may be argued that the disappearance of temporal isolation effects when presentation schedule is unpredictable (rather than either consistently increasing or consistently decreasing) reflects the possibility that attending to a temporal dimension is less useful under such circumstances. By this argument, a temporal dimension might be used when schedule is predictable, even if it is not when presentation is random. The present data go some way to addressing this possibility, at least in the case of free recall, because similar effects of temporal isolation are found whether presentation schedule is predictable or unpredictable.

A second, related point concerns the contrast between serial and free recall. It was noted above that the task requirements of serial recall highlight or even demand the use of a positional dimension in a way that free recall does not and that a task-induced focus on a temporal dimension may distinguish free and serial recall. The present results 
are consistent with this possibility. However, we note that the methodology used in the present free recall experiments differs in a number of ways from that used in many previous studies that have failed to show temporal isolation effects in serial recall. Specifically, the experiments we described above used words (an open pool), rather than letters or digits, as stimuli, used longer lists than are typically used in serial recall tasks, and, in particular, used a continuous distractor methodology, rather than articulatory suppression, to prevent rehearsal. Such methodological differences may yet turn out to be important.

In summary, the present results appear to be consistent with temporal distinctiveness models of free recall (e.g., Brown et al., 2002), mark a contrast between serial and free recall in terms of reliance on temporal distinctiveness, and add indirect support to time-based accounts of recency effects in free recall.

\section{REFERENCES}

BJork, R. A., \& Whitten, W. B. (1974). Recency-sensitive retrieval processes in long-term free recall. Cognitive Psychology, 6, 173-189.

Brown, G. D. A., Neath, I., \& Chater, N. (2002). A ratio model of scale-invariant memory and identification. Unpublished manuscript.

Brown, G. D. A., Preece, T., \& Hulme, C. (2000). Oscillator-based memory for serial order. Psychological Review, 107, 127-181.

Corballis, M. C. (1966a). Memory span as a function of variable presentation speeds and stimulus durations. Journal of Experimental Psychology, 71, 461-465.

Corballis, M. C. (1966b). Rehearsal and decay in immediate recall of visually and aurally presented items. Canadian Journal of Psychology, 20, 43-51.

Crowder, R. G. (1976). Principles of learning and memory. Hillsdale, NJ: Erlbaum.

Davelaar, E. J., Goshen-Gottstein, Y., Ashienazi, A., HaArMANN, H. J., \& UsHeR, M. (2005). The demise of short-term memory revisited: Empirical and computational investigations of recency effects. Psychological Review, 112, 3-42.

Glenberg, A. M., \& Swanson, N. G. (1986). A temporal distinctiveness theory of recency and modality effects. Journal of Experimental Psychology: Learning, Memory, \& Cognition, 12, 3-15.

Henson, R. N. A. (1999). Positional information in short-term memory: Relative or absolute? Memory \& Cognition, 27, 915-927.

Howard, M. W., \& KAHANA, M. J. (1999). Contextual variability and serial position effects in free recall. Journal of Experimental Psychology: Learning, Memory, \& Cognition, 25, 923-941.

Howard, M. W., \& KaHANA, M. J. (2002). A distributed representation of temporal context. Journal of Mathematical Psychology, 46, 269-299.

Kahana, M. J. (1996). Associative retrieval processes in free recall. Memory \& Cognition, 24, 103-109.
LAMING, D. (1999). Testing the idea of distinct storage mechanisms in memory. International Journal of Psychology, 34, 419-426.

Lewandowsky, S., \& Brown, G. D. A. (2005). Serial recall and presentation schedule: A micro-analysis of local distinctiveness. Memory, 13, 283-292.

Lewandowsky, S., Brown, G. D. A., Wright, T., \& Nimmo, L. M. (2006). Timeless memory: Evidence against temporal distinctiveness models of short-term memory for serial order. Journal of Memory \& Language, 54, 20-38.

Nairne, J. S., Neath, I., Serra, M., \& Byun, E. (1997). Positional distinctiveness and ratio rule in free recall. Journal of Memory \& Language, 37, 155-166.

Neath, I. (1993). Distinctiveness and serial position effects in recognition. Memory \& Cognition, 21, 689-698.

Neath, I., \& Crowder, R. G. (1990). Schedules of presentation and temporal distinctiveness in human memory. Journal of Experimental Psychology: Learning, Memory, \& Cognition, 16, 316-327.

NeAth, I., \& Crowder, R. G. (1996). Distinctiveness and very shortterm serial position effects. Memory, 4, 225-242.

NG, H. L. H., \& MAYBERY, M. T. (2002). Grouping in short-term verbal memory: Is position coded temporally? Quarterly Journal of Experimental Psychology, 55A, 391-424.

Nimmo, L. M., \& LEWANDOWSKY, S. (2005). From brief gaps to very long pauses: Temporal isolation does not benefit serial recall. Psychonomic Bulletin \& Review, 12, 999-1004.

Nimmo, L. M., \& Lewandowsky, S. (in press). Distinctiveness revisited: Temporal isolation does not benefit short-term serial recall of heard or seen events. Memory \& Cognition.

RöNNBERG, J. (1980). Predictability as a task demand in single-trial free recall. Scandinavian Journal of Psychology, 21, 83-95.

RöNNBERG, J. (1981). Predictability and recall strategy for nominal serial position curves. Scandinavian Journal of Psychology, 22, 189-195.

TAN, L., \& WARD, G. (2000). A recency-based account of the primacy effect in free recall. Journal of Experimental Psychology: Learning, Memory, \& Cognition, 26, 1589-1625.

Welte, J., \& LaUghery, K. (1971). Short-term memory: The effects of inter-item time distribution and recall procedure. Canadian Journal of Psychology, 25, 436-442.

WILSON, M. (1988). MRC Psycholinguistic Database: Machine-usable dictionary, version 2. Behavior Research Methods, Instruments, \& Computers, 20, 6-10.

\section{NOTES}

1. The analyses were repeated looking at all recalls (i.e., without excluding the case in which the items preceding, or following, a target item were also recalled). The pattern of significant results was unchanged.

2 . The analyses were repeated with a hierarchical linear regression model, to permit estimates of the intercept term, plus the predictors, to vary across participants, while computing the statistical significance of those parameter estimates across all participants. The same pattern of results was obtained.

(Manuscript received August 16, 2005; revision accepted for publication December 12, 2005.) 